\title{
Detection of Unbalanced Voltage Cells in Series-connected Lithium-ion Batteries Using Single-frequency Electrochemical Impedance Spectroscopy
}

\author{
Norihiro Togasaki*, Tokihiko Yokoshima, Yasumasa Oguma, and Tetsuya Osaka* \\ Research Organization for Nano and Life Innovation, Waseda University, 513 Waseda-tsurumaki-cho, Shinjuku-ku, Tokyo \\ 162-0041, Japan
}

\begin{abstract}
For a battery module where single cells are connected in series, the single cells should each have a similar state of charge (SOC) to prevent them from being exposed to an overcharge or over-discharge during charge-discharge cycling. To detect the existence of unbalanced SOC cells in a battery module, we propose a simple measurement method using a single-frequency response of electrochemical impedance spectroscopy (EIS). For a commercially available graphite/nickel-cobaltaluminum-oxide lithium-ion cell, the cell impedance increases significantly below SOC $20 \%$, while the impedance in the medium SOC region (SOC20\%-SOC $80 \%$ ) remains low with only minor changes. This impedance behavior is mostly due to the elementary processes of cathode reactions in the cell. Among the impedance values $\left(Z, Z^{\prime}, Z^{\prime \prime}\right)$, the imaginary component of $Z^{\prime \prime}$ regarding cathode reactions changes heavily as a function of SOC, in particular, when the EIS measurement is performed around $0.1 \mathrm{~Hz}$. Thanks to the significant difference in the time constant of cathode reactions between $\leq \mathrm{SOC} 10 \%$ and $\geq \mathrm{SOC} 20 \%$, a single-frequency EIS measurement enlarges the difference in impedance between balanced and unbalanced cells in the module and facilitates an $\sim 80 \%$ improvement in the detection signal compared to results with conventional EIS measurements.
\end{abstract}

Keywords : Lithium-Ion Battery, Battery Module, Cell Balance, Electrochemical Impedance Spectroscopy, Time Constant Received : 26 January 2021, Accepted : 31 March 2021

\section{Introduction}

Lithium-ion batteries have been widely used in electric vehicles. Because automobiles require a high power density and high capacity, battery cells are typically connected in series and in parallel to form a battery module. To extract as much electrochemical energy as possible over a long period of time from the battery module, each battery cell in the module should be operated within its thermodynamically stable voltage range; that is, it is desirable that the state of charge (SOC) of each single cell in the module should be similar during operation, called cell balance. However, the SOC of each cell in the module

*E-mail address: n-togasaki@ruri.waseda.jp, osakatets@waseda.jp DOI: https://doi.org/10.33961/jecst.2021.00115

This is an open-access article distributed under the terms of the Creative Commons Attribution Non-Commercial License (http://creativecommons.org/licenses/by-nc/4.0) Attribution Non-Commercial License (http://creativecommons.org/licenses/by-nc/4.0)
which permits unrestricted non-commercial use, distribution, and reproduction in any which permits unrestricted non-commercial use, distri
medium, provided the original work is properly cited. can differ slightly during charge-discharge operations, owing to variations in atmospheric temperature or the manufacturing process. Unbalanced SOC cells in the module are potential triggers of an overcharge [1] or an over-discharge [2], which can lead to combustion and explosion. However, these are inherently difficult to identify without external electrical circuits for measuring the voltage of each cell.

To ensure battery safety, a passive or active balance circuit is required in current battery modules; the former consumes extra charge from battery cells with higher SOC using a resistor during charging and the latter redistributes a certain amount of charge from a higher SOC cell to a lower SOC cell during charging and discharging [3]. However, installation of these balancing circuits increases cost and limits freedom in battery design. Moreover, it is difficult to apply such balancing circuits for bipolar structured batteries incorporating solid-state electrolytes [4] because of the limited areas of their very thin electrodes. 
Therefore, it is critically important to develop a measurement technique for diagnosing cell balance in a battery module without external electrical circuits.

Electrochemical impedance spectroscopy (EIS) is one of the most effective methods for understanding the internal status of the battery cell without any cell destruction. In the EIS measurement, a small input signal of voltage or current is loaded into a battery cell and we analyze the output signal using an appropriate equivalent circuit. The frequency of the input signal is typically chosen in the range of several hundred $\mathrm{kHz}-10 \mathrm{mHz}$. This allows us to separate electrochemical reactions that take place in the battery cell into elementary processes on the basis of the different time constant. It is generally known that the impedance of each elementary process in battery cells changes significantly as a function of SOC $[5,6]$, thereby, the cell balance in the series-connected cells can be detected precisely using EIS measurement. However, a full-scale sweep of frequencies and data analysis takes a long time in the conventional impedance measurement/analysis. Therefore, more simple and short-time measurements are required in the practical application such as for the installation into battery management systems. Single-frequency impedance spectroscopy is a potential option for solving this dilemma. Several reports demonstrated that an impedance value at a specific frequency of $316 \mathrm{~Hz} / 500 \mathrm{~Hz}$ which corresponds to the reaction of solid electrolyte interphase on anode is a good indicator to understand the state of battery health in lithium cobalt oxide (LCO)/carbon systems [7,8]. Meanwhile, in many cases in lithium-ion batteries, the impedance of cathode reactions of high-capacity materials such as LCO [9] and nickel manganese cobalt oxide (NMC) [6] is more dominant in a wide SOC range compared to anode reactions and the cathode impedance accounts for a large part of the entire impedance of the cell. Aurbach et al. reported that the impedance of transition metal oxide cathodes $\left(\mathrm{Li}_{\mathrm{x}} \mathrm{NiO}_{\mathrm{y}}, \mathrm{Li}_{\mathrm{x}} \mathrm{MnO}_{\mathrm{y}}\right)$ obviously show potential dependent behavior [10] while graphite electrodes are potential-independent [11]. Therefore, focusing on cathode impedance and its variation as a function of SOC could be an advantageous way to detect cellbalance in series-connected cells.

This paper reports a simple method to detect the presence or absence of unbalanced SOC cells in series-connected batteries using single-frequency
EIS. In this study, we focus on a dominant electrochemical process (cathode reactions) in the cell with an impedance that dramatically changes as a function of SOC. It demonstrates that the impedance $Z$ " obtained at a specific frequency can be a good indicator of the cell balance of series-connected batteries. The novelty of this study how to choose an appropriate frequency for impedance measurement is described in the literature.

\section{Experimental}

High-energy 18650 type cylindrical cells with a capacity of $\sim 2.9$ A $\cdot h$ were used throughout this study. The anodes and cathodes in the cells are composed of graphite and nickel cobalt aluminum oxide (NCA), whose details have been investigated in our previous paper [1].

For the measurement of the impedance of a single cell, the cell voltage was set to $3.00-4.20 \mathrm{~V}$, which corresponds to SOC $0 \%-\mathrm{SOC} 100 \%$, and the impedance spectra of the battery cells were measured using a potentiostat (CellTest System 1470E, Solartron) and a frequency analyzer (CellTest System 1400, Solartron). We used four battery cells to prepare series-connected cells. The cell voltage of each cell was set to SOC $10 \%-\mathrm{SOC} 60 \%$ and the total voltages of the prepared series-connected cells were 14.714.8 V (Group A), 14.4-14.5 V (Group B), and 13.8$13.9 \mathrm{~V}$ (Group C). The impedance spectra of the series-connected cells were measured using ModuLab XM ECS (Solartron). Throughout this study, impedance measurements were performed at $25^{\circ} \mathrm{C}$ at the open-circuit voltage with a $\sim 10 \mathrm{mV}$ (zero to peak) AC signal per cell in a frequency range of $10 \mathrm{kHz}$ to $10 \mathrm{mHz}$.

We designed the equivalent circuit shown in Fig. 2 (a) for analyzing the obtained impedance spectra. The equivalent circuit contains $\mathrm{R}_{\mathrm{I}}$ (resistance of the battery leads and connected cable for impedance measurement), $R_{S}$ (resistance of the electrolyte in the cell), $\mathrm{R}_{\mathrm{CT}}$ (charge transfer resistance of the anode $\left(\mathrm{R}_{\mathrm{CT}-\mathrm{A}}\right)$ and cathode $\left(\mathrm{R}_{\mathrm{CT}-\mathrm{C}}\right)$ in the cell, respectively) and CPE (constant phase element of the anode $\left(\mathrm{CPE}_{\mathrm{A}}\right)$ and cathode $\left(\mathrm{CPE}_{\mathrm{C}}\right)$ electrode surfaces, respectively). We used the constant phase element (CPE) to express the capacitive behavior between electrode and electrolyte, which is generally used for the squashed semi-circle caused by the non-uniform 
electrochemical reactions on the electrode surface [12]. The interfacial capacitance $\left(\mathrm{C}_{\text {int }}\right)$ between electrode and electrolyte can be calculated by the following equation:

$$
C_{\text {int }}=\frac{1}{2 \pi \times R_{C T} \times f_{\max }}
$$

Where $\mathrm{R}_{\mathrm{CT}}$ is the charge transfer resistance of anode or cathode, and $f_{\max }$ is the apex frequency of the capacitive semicircle in anode or cathode. $f_{\max }$ can be obtained by the data fitting by following procedure.

Data fitting was performed using the Microsoft Excel Solver package until minimum error was obtained. This was achieved by minimizing the sums of all differences between the complex impedance data acquired from experiments and the impedances calculated from the equivalent circuit using the fitted parameters at each frequency, similar to the procedure detailed in our previous reports [5].

\section{Results and Discussion}

Fig. 1 shows Nyquist plots obtained from the graphite/NCA lithium-ion battery cells with SOCs ranging from $0 \%$ to $100 \%$. Two capacitive semi-circles (half circles) appear in the high-frequency region and the low-frequency region, suggesting that there are at least two elemental electrochemical processes in the battery cell. Compared to the semi-circle in the high-frequency region, the semi-circle in the low-frequency region changes heavily as a function of SOC.

To attribute those two capacitive semi-circles with elementary electrochemical processes in the cell, the Nyquist plots in Fig. 1 are analyzed using the equivalent circuit shown in Fig. 2(a). It has two parallel connections of resistance and capacitance corresponding to the anode and cathode, with series connections of resistor $\left(\mathrm{R}_{\mathrm{I}}\right)$ from the wiring and measuring equipment and of the solution resistance $\left(\mathrm{R}_{\mathrm{S}}\right)$. To ignore the contribution of the inductor component, which is observed in the high-frequency region $(>1 \mathrm{kHz})$ in the fourth quadrant, impedance fitting was performed below $1 \mathrm{kHz}$. Details of each component in the equivalent circuit are written in the Experimental section. Fig. 2(b) shows the apex frequency of the capacitive semi-circles with respect to the elemental electrochemical processes in the battery cell as a function of SOC. The apex frequency in the capacitive semi-circle in the low-frequency region (red dots) changes heavily with SOC compared to that in the high-frequency region (blue dots); the apex frequency in the low-frequency region becomes lower in the low SOC range of $\angle \mathrm{SOC} 40 \%$ and in the high

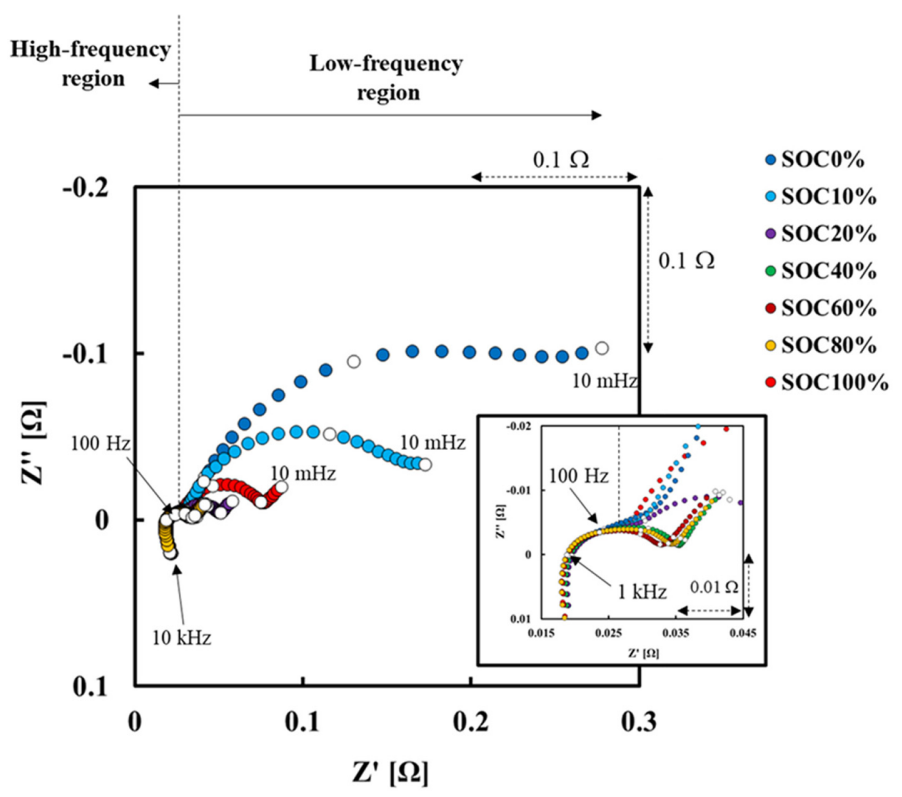

Fig. 1. Nyquist plots from the graphite/NCA lithium-ion cells with SOCs set to be SOC $0 \%$, SOC $10 \%$, SOC $20 \%$, SOC $40 \%$, SOC $60 \%$, SOC $80 \%$, and SOC $100 \%$. The inset figure shows a magnification of the high-frequency region. 
(a)

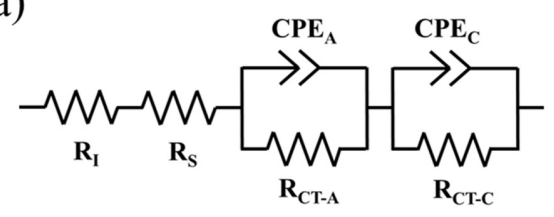

(b)

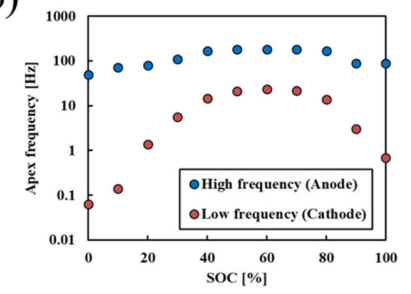

(d)

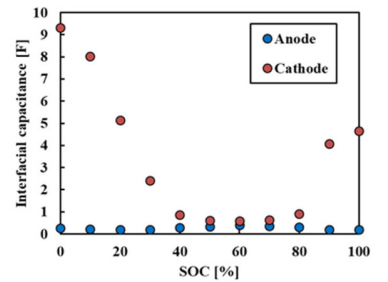

(c)

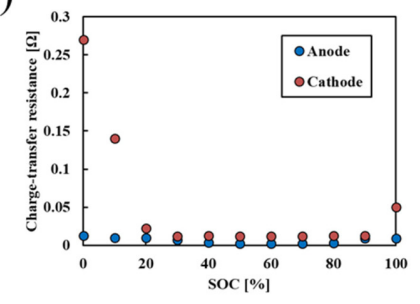

(e)

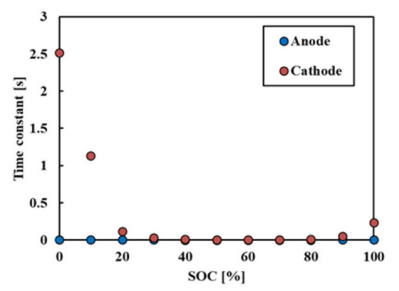

Fig. 2. (a) Equivalent circuit used to analyze the impedance spectra in this study. $\mathrm{R}_{\mathrm{I}}$ : resistance of the battery leads and connected cable; $\mathrm{R}_{\mathrm{S}}$ : resistance of the electrolyte; $\mathrm{R}_{\mathrm{CT} \text {-A }}$ and $\mathrm{R}_{\mathrm{CT} \text {-C }}$ : charge transfer resistance of the anode and cathode, respectively; and $\mathrm{CPE}_{\mathrm{A}}$ and $\mathrm{CPE}_{\mathrm{C}}$ : constant phase element of the anode and cathode electrode surfaces, respectively. Fitting results of the (b) apex frequencies of the capacitive semi-circles, (c) charge-transfer resistance, (d) interfacial capacitance, and (e) time constants with respect to the anode and cathode.

SOC region of $>$ SOC $80 \%$. This behavior is similar to the results obtained from a NMC cathode by Ovejas et al., who reported that the apex frequency of the $\mathrm{NMC}$ cathode changes over $2-28 \mathrm{~Hz}$ for SOC $10 \%-$ SOC $100 \%$, and is low for low $(<$ SOC $40 \%)$ and high ( $>$ SOC $80 \%$ ) SOCs [6]. In our previous study, where activation energy was calculated using the same battery cell, we reported that the semi-circle with an apex frequency near $1 \mathrm{~Hz}$ at SOC $100 \%$ can be attributed to NCA cathode reactions [1]. Therefore, the semi-circle in the low-frequency region can be attributed to NCA cathode reactions.

Except for cathode reactions, the elementary processes of the graphite anode and solid electrolyte interphase (SEI) on the anode should be taken into consideration in the cell. The apex frequency in the semi-circle at high frequency changes between $50 \mathrm{~Hz}$ and $180 \mathrm{~Hz}$ in the measured SOC range. Gordon et $a l$. reported that the apex frequency of the charge transfer resistance in the graphite electrode was 40 hertz at $25^{\circ} \mathrm{C}$ [13]. By contrast, the apex frequency corresponding to SEI is typically observed at a few hundred hertz $[6,14]$. We previously reported that the capacitive semi-circles of the graphite anode and SEI overlapped each other at temperatures of $20^{\circ} \mathrm{C}[1,15]$. Therefore, the semi-circle in the high-frequency region is attributed to the anode reactions, which combine signals from the graphite and SEI.

The results above confirm that the semi-circles at the low and high frequencies correspond to cathode and anode reactions, respectively. Fig. 2(c) shows the fitting results of the charge transfer resistance of the NCA cathode $\left(R_{\text {CT-C }}\right)$ and graphite anode $\left(R_{\text {CT-A }}\right)$. Compared to $\mathrm{R}_{\mathrm{CT}-\mathrm{A}}, \mathrm{R}_{\mathrm{CT} \text { - }}$ changes dramatically as a function of SOC; in particular, a remarkable increase was observed in the region of $<$ SOC $20 \%$. This may be due to the low electronic conductivity of active materials of the NCA cathode during the early stage 
of deintercalation; the conductivity of the layered cathode active materials such as lithium cobalt oxide $\left(\mathrm{Li}_{1-x} \mathrm{CoO}_{2}\right)$ decreases drastically at lower SOC regions (where $x$ changes from 0 to 0.1 ), owing to the metal-insulator transition $[16,17]$. Similar behavior was reported by Nobili et al. in the mixed oxides of $\mathrm{Li}_{x} \mathrm{Ni}_{0.8} \mathrm{Co}_{0.2} \mathrm{O}_{2}$ [18], which may account for the observed increased impedance in $\mathrm{R}_{\mathrm{CT}-\mathrm{C}}$ at $<\mathrm{SOC} 20 \%$ in Fig. 2(c). Similar behavior depending on the SOC was observed in the interfacial capacitance of the cathode in Fig. 2(d); a stronger dependence on SOC was observed in the cathode compared to that in the anode. The interfacial capacitance was calculated using the equation in the Experimental section. The interfacial capacitance of the cathode changes significantly below SOC $40 \%$ or above SOC $80 \%$, while the capacitance of the anode does not change considerably in the range of SOC $0 \%-S O C 100 \%(0.09-$ $0.15 \mathrm{~F}$ ). The result that lower and higher SOCs show higher capacitance is similar to the results for the layered cathode active material of LCO-NMC [6].

The above results indicate that the strong SOC dependence can be more clearly observed in the charge-transfer resistance and interfacial capacitance of the NCA cathode. In the same manner, a strong SOC dependence of the time constant, which is calculated from the interfacial capacitance multiplied by the charge-transfer resistance, was seen in the NCA cathode with a significant increase in the SOC region below 20\% (Fig. 2(e)).

For a graphite/NCA lithium-ion battery, a strong potential dependence on SOC of the impedance in the elementary process of the NCA cathode was confirmed. This phenomenon is typically observed in layered cathode materials, as reported by Aurbach et al. [10], while the graphite anode exhibits potentialindependent behavior [11]. In particular, it varies dramatically in the low SOC region of $<$ SOC $20 \%$. Fig. 3 shows contour maps of the impedance of $Z, Z^{\prime}$, and $Z^{\prime \prime}$ of the graphite/NCA lithium-ion cell in the frequency range of $31.62-0.032 \mathrm{~Hz}$ as a function of SOC (SOC10\%-SOC $80 \%$ ). Regardless of Z, Z', and $Z^{\prime \prime}$, it can be seen that the impedance value in the low-frequency range $(<1 \mathrm{~Hz})$ varies heavily with the SOC as compared to the high-frequency band $(>1$ $\mathrm{Hz}$ ). The impedances of $Z$ and $Z^{\prime}$ exhibit similar behavior. Compared to $Z$ and $Z^{\prime}$, the impedance parameter of $Z$ " exhibits unique SOC dependence. Note that the impedance of $Z^{\prime \prime}$ around $0.13 \mathrm{~Hz}$ in the
SOC region of $\geq$ SOC $20 \%$ has an extremely small value with no significant SOC dependence, while it increases rapidly in the region of $<\mathrm{SOC} 20 \%$. At $0.13 \mathrm{~Hz}$, the impedance of $\mathrm{Z}^{\prime \prime}$ at SOC $10 \%$ is over 10 times higher than that at SOC $20 \%$, which is a larger difference than that observed between SOC $10 \%$ and SOC20\% in $\mathrm{R}_{\text {CT-C }}$ and the interfacial capacitance of the cathode. This suggests that the impedance at a single frequency enlarges the SOC dependence in impedance $Z$ ", because of the usage of a significant change of $\mathrm{R}_{\mathrm{CT}-\mathrm{C}}$ (Fig. 2(c)) and the reaction time constant (Fig. 2(e)) between SOC $10 \%$ and $\geq$ SOC $20 \%$.

As is shown in Figs. S1(a) and (b), small values of $Z^{\prime \prime}$ at $>$ SOC20\% correspond to the result that $Z^{\prime \prime}$ at $0.13 \mathrm{~Hz}$ located near the low-frequency end of the NCA semi-circle (or the starting point of diffusion impedance) in the Nyquist plots, where they are close to the real axis. By contrast, a high value of $Z^{\prime \prime}$ at $<$ SOC20\% corresponds to the result that $Z$ " at $0.13 \mathrm{~Hz}$ is located near the apex frequency of the semi-circle in the NCA cathode in the Nyquist plots. In short, the impedance rise of $\mathrm{R}_{\mathrm{CT}-\mathrm{C}}$ from $\geq \mathrm{SOC} 20 \%$ to SOC $10 \%$ enlarges the diameter of the NCA cathode's semi-circle. In addition, the phase angle in the impedance at a certain frequency $(0.13 \mathrm{~Hz}$ in this study) appears to increase from $\geq \mathrm{SOC} 20 \%$ to SOC $10 \%$, owing to the decrease of the reaction time constant in the NCA cathode, resulting in a remarkable difference in impedance $Z^{\prime \prime}$ (Fig. 3(d)).

Consequently, the following procedure can be proposed to identify the impedance change in the low SOC region.

- Perform EIS and focus on the dominant elemental process (which in this study is a semi-circle at low frequency) in the cell impedance (see Fig. 1).

- Perform fitting analysis for the obtained Nyquist plots to understand the SOC range where the impedance for the dominant elemental process is small (which in this study is SOC40\%-SOC $80 \%$ ) (see Fig. 2).

- In the above case, choose the lowest frequency at the end of the capacitive semi-circle in the dominant reaction process (which in this study is approximately $0.13 \mathrm{~Hz}$ ) (see Fig S1).

- Perform single-frequency EIS at the obtained frequency $(0.13 \mathrm{~Hz}$ ) (see Fig. 3).

From the results above, it can be seen that the value of $Z^{\prime \prime}$ is a good indicator of an SOC difference in a single cell. The key point is to choose an appropriate 
frequency to enlarge the difference of $Z^{\prime \prime}$ depending on the cell's SOC.
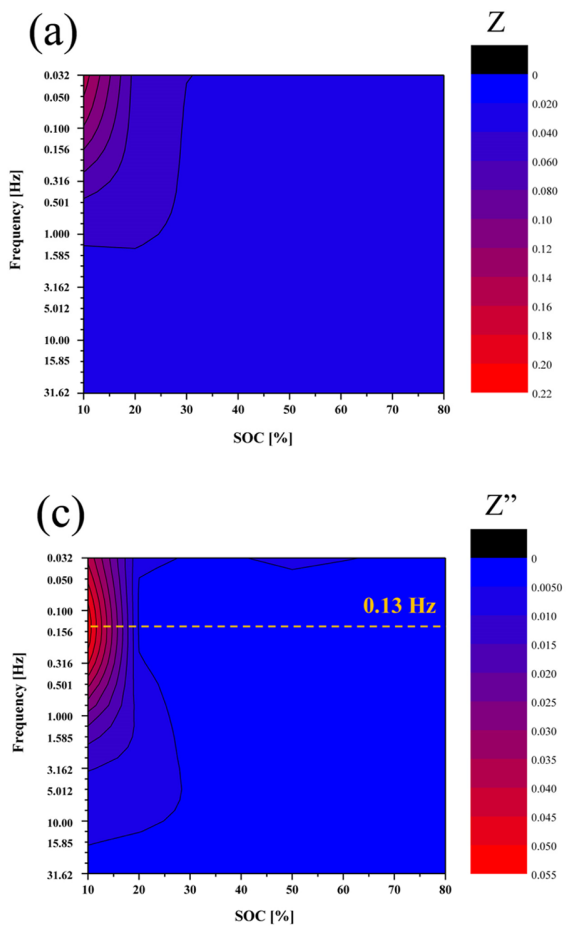

As Fig. S2 indicates, the total impedance of seriesconnected cells corresponds to the sum of the imped-

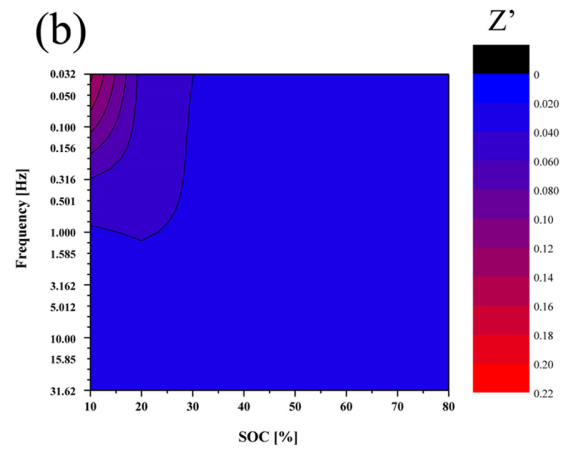

(d)

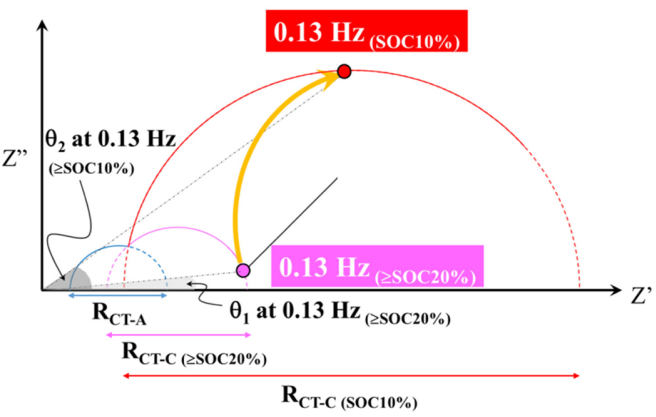

Fig. 3. Contour maps of the impedances of (a) $Z$, (b) $Z^{\prime}$, and (c) $Z^{\prime \prime}$ in the frequency range of $31.62-0.032 \mathrm{~Hz}$ as a function of the SOC of the graphite/NCA lithium-ion cell in the range of SOC $10 \%-\mathrm{SOC} 80 \%$. (d) Schematic of the increase of impedance $Z^{\prime \prime}$ at $0.13 \mathrm{~Hz}$ between $\geq \mathrm{SOC} 20 \%$ and $\mathrm{SOC} 10 \%$. Unlike the behavior of $\mathrm{R}_{\text {CT-A }}$, a significant change in $\mathrm{R}_{\text {CT-C }}$ and the reaction time constant of the NCA cathode between $\geq \mathrm{SOC} 20 \%$ and SOC $10 \%$ enlarges the difference in impedance $Z^{\prime \prime}$ at $0.13 \mathrm{~Hz}$.

Table 1. Nine experimental conditions for the four series-connected cells evaluated in this study. Group A, Group B, and Group $\mathrm{C}$ are categorized as having similar voltage conditions. In each group, the condition No. 1 shows the SOCs in four battery cells are identical to be each other. The condition No. 2 and No. 3 show some SOC variations of single cells in series-connected cells; the SOC variation (= maximum SOC - minimum SOC) in the condition No. 2 is SOC $20 \%$ and that in the condition No. 3 is SOC $30 \%$.

\begin{tabular}{ccllllc}
\hline \hline No. & Group & & SOC [\%] & & Voltage [V] \\
\hline 1 & A & 40 & 40 & 40 & 40 & 14.7 \\
2 & A & 30 & 40 & 40 & 50 & 14.7 \\
3 & A & 30 & 30 & 50 & 60 & 14.8 \\
1 & B & 30 & 30 & 30 & 30 & 14.4 \\
2 & B & 20 & 30 & 30 & 40 & 14.4 \\
3 & B & 20 & 20 & 40 & 50 & 14.5 \\
1 & C & 20 & 20 & 20 & 20 & 13.9 \\
2 & C & 10 & 20 & 20 & 30 & 13.8 \\
3 & C & 10 & 10 & 30 & 40 & 13.8 \\
\hline
\end{tabular}


(a) Group A

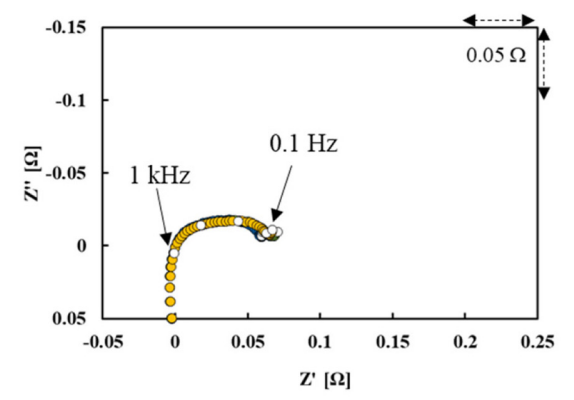

(c) Group C

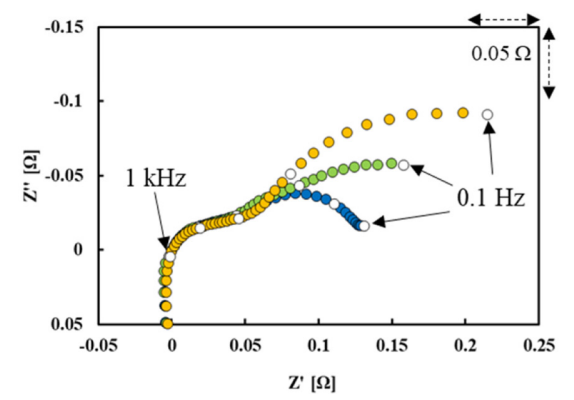

(b) Group B

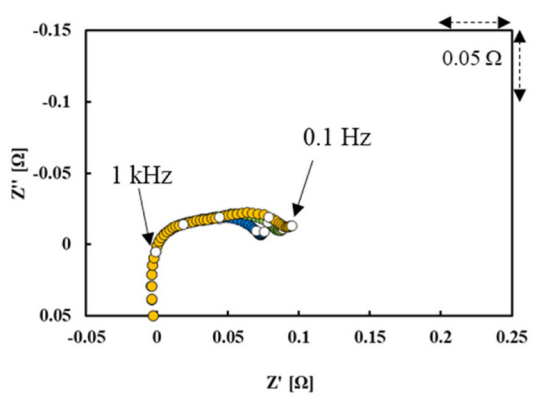

(d)

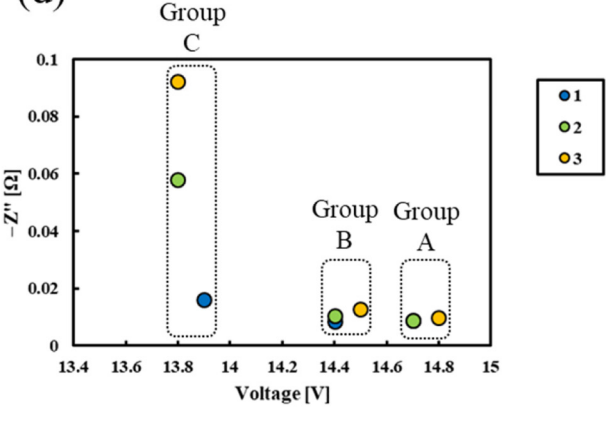

Fig. 4. Nyquist plots obtained from the series-connected cells with voltage set to (a) 14.7-14.8 V (Group A), (b) 14.414.5 V (Group B), and (c) 13.8-13.9 V (Group C) and measured for the frequency range from $10 \mathrm{kHz}$ to $0.1 \mathrm{~Hz}$. (d) The results for extracted Z" values at $0.13 \mathrm{~Hz}$ for the voltages of Group A, Group B, and Group C.

ances from individual cells. Therefore, enlargement of a single cell's impedance depending on the SOC in the battery module is critically important for detecting SOC variation in the module. To detect the presence of unbalanced SOC cells in series-connected cells, impedance measurements were performed for the nine conditions shown in Table 1 . The total module voltage is categorized into three groups of 14.714.8 V (Group A), 14.4-14.5 V (Group B), and 13.8$13.9 \mathrm{~V}$ (Group C), and each module incorporates four battery cells with different SOCs.

Figs. 4(a)-(c) show the results obtained for the frequency range from $10 \mathrm{kHz}$ to $0.1 \mathrm{~Hz}$. For the Nyquist plots in Figs. 4(a)-(c), the impedances of $R_{I}$ and $R_{S}$ were subtracted to simplify the obtained results. Fig. 4(d) shows the results for the extracted $Z$ " value for each condition at $0.13 \mathrm{~Hz}$. For Fig. 4(d), there is no significant difference between the conditions for impedance $Z$ " when the total voltage is set to 14.7 14.8 V (Group A) and to 14.4-14.5 V (Group B), suggesting that it is difficult to detect the SOC varia- tion of single cells in series-connected cells when the total voltage of the module is set to $14.7-14.8 \mathrm{~V}$ (Group A) and 14.4-14.5 V (Group B). This is because the cells in the module, which have SOC20\%-SOC60\%, exhibit a similar impedance Z" at $0.13 \mathrm{~Hz}$ (Fig. 3).

By contrast, there is a large difference in impedance in Group C for a total voltage of 13.8-13.9 V; the impedance of the four series-connected cells including the SOC $10 \%$ cell (No. 2 and No. 3 ) is higher than that of the excluded SOC $10 \%$ cell (No. 1), and the impedance increases with an increase in the number of SOC $10 \%$ cells in the series-connected cells. As shown in Fig. 3(c), these results reflect the fact that the impedance $Z$ " of SOC $10 \%$ at $0.13 \mathrm{~Hz}$ is significantly larger than the impedance $Z^{\prime \prime}$ of $\geq$ SOC $20 \%$ at $0.13 \mathrm{~Hz}$. The impedances at each frequency for the three conditions in Fig. 4(c) can be seen to be different from each other. Indeed, the $\mathrm{R}_{\mathrm{CT} \text {-C }}$ and the interfacial capacitance of the cathode in No. 3 can be calculated to be, respec- 
tively, 3.1 times and 3.8 times as high as those in No. 1 via a fitting analysis. However, a more significant difference is obtained by comparing impedances with single-frequency EIS; the value of $Z^{\prime \prime}$ in No. 3 is 5.7 times as high as that in No. 1 , which is an $\sim 80 \%$ improvement for the detection signals in the presence or absence of unbalanced cells in the battery module. This result confirms that single-frequency EIS at a specific frequency enlarges the SOC dependence of impedance, regardless of whether it is a single cell or module.

Since the total impedance of series-connected cells corresponds to the sum of the impedance from individual cells, the relationship between the total impedance and the number of the cells in series can be simply calculated. Fig. S3 (a) and (b) show the impedance $Z^{\prime \prime}$ in Group C (Fig. 4) with a variation of cell numbers in series connected cells. The information of SOCs and cell numbers are written in Table $\mathrm{S} 1$. The error bar shown in Fig. S3 (a) and (b) suggests the dispersion of impedance $Z^{\prime \prime}( \pm 3 \sigma)$ which is calculated by using the data from 10 individual battery cells in the same product lot (Fig. S3 (c)). Fig. S3 (a) and (b) clearly show that three conditions of No. 1, No.2, and No.3 are observed to be different to each other regardless of cell numbers in series, suggesting that the present analytical method is applicable for large-scale battery systems. We believe these results provide a new insight for diagnosing SOC cell balance in battery modules without external electrical circuits.

\section{Conclusions}

In this study, we proposed a simple measurement method using single-frequency electrochemical impedance spectroscopy (EIS) to detect cells with an unbalanced state of charge (SOC) in a series-connected battery module. For a graphite/nickel-cobaltaluminum-oxide (NCA) lithium-ion cell, the impedance of the electrochemical process of the cathode changes considerably as a function of cell SOC, in particular, between SOC10\% and SOC $20 \%$, owing to the behavior of electrical conductivity in NCA.

Among the impedance values ( $Z, Z^{\prime}$, and $\left.Z^{\prime \prime}\right)$, the imaginary component of $Z^{\prime \prime}$ corresponding to a frequency regarding cathode reactions changes significantly as a function of SOC because the cathode's charge-transfer resistance and interfacial capacitance change simultaneously, leading to a huge change in the reaction time constant.

Single-frequency EIS at an appropriate frequency with varying the total voltage of the battery module facilitates detection of the presence or absence of unbalanced SOC cells in the module, thanks to the significant impedance change, especially $Z^{\prime \prime}$, of single cell as a function of SOC, and this method achieves the detection of unbalanced SOC cells, with an $\sim 80 \%$ improvement in the detection signals compared to conventional EIS measurement.

This study confirms that cathode and anode active materials that have large impedance changes as a function of SOC with impedance measured in an appropriate frequency region is a potential option for evaluating cell balance in a battery module. Further analysis in terms of using different cathode materials (i.e. NMC and LCO) is ongoing in current research. We believe that this can ensure battery safety and it can be used in bipolar structured batteries where external balancing circuits are difficult to attach.

\section{Author Contributions}

N. T. performed and analyzed all the experiments reported in this manuscript. N. T and T. Y participated in the conception of this study and designed the experiments. Y. O participated in the conception of this study. N. T. wrote the paper. All authors contributed to the discussion and interpretation of the results. This study was supervised by T. O.

\section{Supporting Information}

Supporting Information is available at https:// doi.org/10.33961/jecst.2021.00115

\section{Conflicts of interest}

The authors declare no conflicts of interest.

\section{References}

[1] N. Togasaki, T. Yokoshima, Y. Oguma and T. Osaka, $J$. Power Sources, 2020, 461, 228168.

[2] M. S. Wu, C. Y. Lin, Y. Y. Wang, C. C. Wan and C. R. Yang, Electrochimica Acta., 2006, 52(3), 1349-1357.

[3] M. M. Hoque, M. A. Hannan, A. Mohamed and A. Ayob, Renew. sustain. energy rev., 2017, 75, 1363-1385.

[4] Y. Kato, K. Kawamoto, R. Kanno and M. Hirayama, 
Electrochemistry, 2012, 80(10), 749-751.

[5] T. Osaka, T. Momma, D. Mukoyama, H. Nara, J. Power Sources., 2012, 205, 483-486.

[6] V. J. Ovejas and A. Cuadras, Batteries, 2018, 4(3), 43.

[7] C. T. Love, M. B. V. Virji, R. E. Rocheleau and K. E. Swider-Lyons, J. Power Sources., 2014, 266, 512-519.

[8] C. T. Love and K. Swider-Lyons, Electrochem Solid State Letters., 2012, 15(4), A53.

[9] H. Nara, D. Mukoyama, T. Yokoshima, T. Momma and T. Osaka, J. Electrochem. Soc., 2015, 163(3), A434.

[10] D. Aurbach, K. Gamolsky, B. Markovsky, G. Salitra, Y. Gofer, U. Heider, R. Oesten and M. Schmidt, $J$. Electrochem. Soc., 2000, 147(4), 1322.

[11] M. D. Levi, G. Salitra, B. Markovsky, H. Teller, D. Aurbach, U. Heider and L. Heider, J. Electrochem. Soc., 1999, 146(4), 1279.
[12] M. E. Orazem and B. Tribollet, ChemTexts, 2020, 6(2), $1-9$.

[13] I. A. J. Gordon, S. Grugeon, H. Takenouti, B. Tribollet, M. Armand, C. Davoisne, A. Debart and S. Laruelle, Electrochimica Acta, 2017, 223, 63-73.

[14] T. P. Heins, N. Harms, L. S. Schramm and U. Schroder, Energy Technol., 2016, 4(12), 1509-1513.

[15] T. Momma, M. Matsunaga, D. Mukoyama and T. Osaka, J. Power Sources., 2012, 216, 304-307.

[16] T. Bak, J. Nowotny, M. Rekas, C. C. Sorrell and S. Sugihara, Ionics., 2000, 6(1), 92-106.

[17] M. Shibuya, T. Nishina, T. Matsue and I. Uchida, $J$. Electrochem. Soc., 1996, 143(10), 3157-3160.

[18] F. Nobili, F. Croce, B. Scrosati and R. Marassi, Chem. Mater., 2001, 13(5), 1642-1646. 